\title{
Determination of Ethanol in Blood Samples Using Partial Least Square Regression Applied to Surface Enhanced Raman Spectroscopy
}

\author{
Güneş Açikgöz' ${ }^{1}$ Berna Hamamci' and Abdulkadir Yildiz ${ }^{2}$ \\ ${ }^{1}$ Hatay Vocational School of Health Services, Mustafa Kemal University, Hatay, Turkey \\ ${ }^{2}$ Department of Physics, Science Faculty, Kahramanmaraş Sütçü Imam University, Kahramanmaraş, Turkey
}

\begin{abstract}
Alcohol consumption triggers toxic effect to organs and tissues in the human body. The risks are essentially thought to be related to ethanol content in alcoholic beverages. The identification of ethanol in blood samples requires rapid, minimal sample handling, and non-destructive analysis, such as Raman Spectroscopy. This study aims to apply Raman Spectroscopy for identification of ethanol in blood samples. Silver nanoparticles were synthesized to obtain Surface Enhanced Raman Spectroscopy (SERS) spectra of blood samples. The SERS spectra were used for Partial Least Square (PLS) for determining ethanol quantitatively. To apply PLS method, $920 \sim 820 \mathrm{~cm}^{-1}$ band interval was chosen and the spectral changes of the observed concentrations statistically associated with each other. The blood samples were examined according to this model and the quantity of ethanol was determined as that: first a calibration method was established. A strong relationship was observed between known concentration values and the values obtained by PLS method $\left(R^{2}=1\right)$. Second instead of then, quantities of ethanol in 40 blood samples were predicted according to the calibration method. Quantitative analysis of the ethanol in the blood was done by analyzing the data obtained by Raman spectroscopy and the PLS method.
\end{abstract}

Key words: Blood samples, Raman spectroscopy, Surface Enhanced Raman Spectroscopy, Silver nanoparticles

\section{INTRODUCTION}

Alcohol drinking is a major correlate of health and disease and alcohol related to health problems is common universally. Especially illegally produced and distributed alcohols have a great risk for human health, since they include high quantity of toxic substances $(1,2)$.

Alcohol consumption triggers toxic effect to organs and tissues in the human body. The risks are essentially thought to be related to ethanol content in alcoholic beverages (3). Ethanol is metabolized and rapidly distributed in body soon after consumption. Ethanol intake and its concentration in the body can be determined by the analysis of dif-

Correspondence to: Güneş Açikgöz, Hatay Vocational School of Health Services, Mustafa Kemal University, Hatay, Turkey

E-mail: gunesani@hotmail.com

This is an Open-Access article distributed under the terms of the Creative Commons Attribution Non-Commercial License (http:// creativecommons.org/licenses/by-nc/3.0) which permits unrestricted non-commercial use, distribution, and reproduction in any medium, provided the original work is properly cited. ferent biological fluids (4). Thus, there are many methods to determine alcohol intake in biological fluids and tissues. These results may concern toxicological and forensic chemistry tests, and thus they need to be both precise and rapid (5).

Raman spectroscopy is very useful in biological samples due to advantages such as enabling rapid identification of a compound, ease of use, minimal sample handling, and non-destructive analysis. Raman spectroscopy is frequently used methods in the medical and forensic sciences to determine or identify the molecular structures of the materials from their own vibrational spectra. Obtained vibration spectrum allows the identification and interpretation of the analyte. Furthermore, the use of water as solvent in Raman spectroscopy offers a great advantage (6-9). It can be interpreted in terms of the presence or absence of the existence of functional groups in the spectra obtained from a piece of evidence or from certain unknown samples. It is known that two different undesirable conditions may occur when biological samples are analyzed with Raman spectroscopy (10). These are the fluorescence emission and the weak 
Raman signals, respectively, which must be removed. To remove these undesirable cases, first the fluorescence emission should be exceeded such that a laser light source is required at 785, 830 or $1,064 \mathrm{~nm}$ long wave lengths for the visible near infrared region of the electromagnetic spectra (10). Then, SERS (Surface Enhanced Raman Spectroscopy) method can be considered removing weak Raman signals. The use of metal nanoparticles for the SERS method leads to an increase in weak signals in Raman scattering, and the undesirable second condition can be clear by using nanoparticles. Raman scattering enhanced has emerged as a consequence of the transfer of charge between the molecule and the nanoparticle and localized surface plasmons formed on the metal surface. These effects are known as chemical and electromagnetic enhanced, respectively. Nanoparticles provide significant surface enhanced through Surface Plasmon Resonance (SPR). Gold ( $\mathrm{Au}$ ) and silver $(\mathrm{Ag})$ are the most notable especially within the metal nanoparticles because they can be synthesized in a controlled manner. For this reason, weak signals in Raman scattering obtained in some applications are strengthened by SERS method. Au and Ag are the most widely used nanoparticles for the SERS method. There are different methods for the synthesis of Ag and Au nanoparticles, and nanoparticles with different shapes such as spheres, cubes, prisms, nanobots can be synthesized (11-13). The use of differently shaped nanoparticles for the SERS method leads to an increase in weak signals in Raman scattering.

This study aims to apply Raman Spectroscopy for identification ethanol in blood samples. Silver colloidal nanoparticles were synthesized to obtain SERS spectra of blood samples. The SERS spectra were used for Partial Least Square (PLS) for determining ethanol quantitatively.

\section{MATERIALS AND METHODS}

Synthesis of AgNPs. The SERS spectra were acquired using two types of aqueous colloidal suspensions of metal nanoparticles (NP) as nanostructured substrates: citratereduced AgNPs. AgNPs were synthesized according to Lee and Meisel method (14).

Chemicals were supplied from Sigma Aldrich (St. Louis MO, USA). UV/Visible spectrophotometer and Scanning Electron Microscopy (SEM/EDS) analysis unit (JEOL 5500/OXFORD Inca-X) were used to characterize AgNPs.

Preparation of blood samples. In order to obtain blood samples prior to research, ethics committee and voluntary approval form was taken from the Ethics Committee of our university, and the experimental methods are in accordance with the approved guidelines.

Attention was paid to take blood samples to be analyzed ethanol, storing them, and delivering them to the laboratory. Blood samples were taken from 10 persons who were

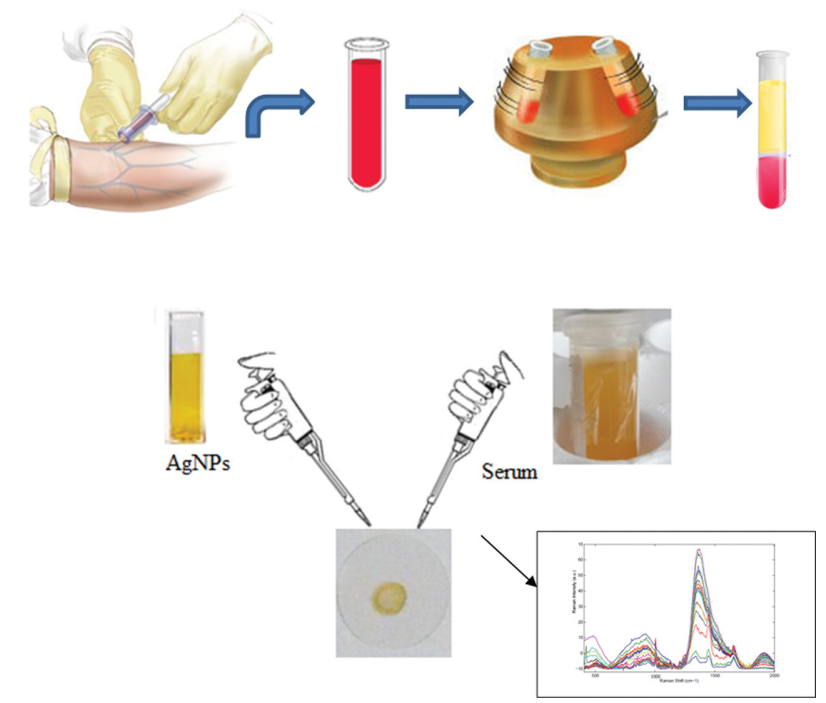

Fig. 1. Preparation of blood samples. Peripheral blood samples were taken $3 \mathrm{~mL}$ into NaF-containing biochemical tubes as fixing agent to prevent alcohol loss. Blood samples were centrifuged at 3,500 rpm for 5 min serum is obtained by removing supernatant. Before SERS measurement, $20 \mu \mathrm{L}$ of serum was mixed with $20 \mu \mathrm{L}$ AgNPs. The mixture is stirred with the pipette on $\mathrm{CaF}_{2}$ slide tip to make it as homogeneous as possible. Then the mixture was incubated for $1 \mathrm{hr}$ at room temperature. Finally, a drop of this mixture was transferred onto an aluminum plate and air dried for SERS measurement.

brought to the official health institution by coma or different reasons and in whose blood was found to high levels of ethanol. It is known that the levels of ethanol in these samples were measured by Gas Chromatography (GC) and ranged from 232 to $386.8 \mathrm{mg} / \mathrm{dL}$. These samples were used for calibration. Blood samples were collected from total 40 individuals, consisting of 20 people who use alcohol and 20 people who do not use alcohol, for predict to quantity of ethanol. Peripheral blood samples were taken $3 \mathrm{~mL}$ into NaF-containing biochemical tubes as fixing agent to prevent alcohol loss. Blood samples were centrifuged at $3,500 \mathrm{rpm}$ for $5 \mathrm{~min}$. serum is obtained by removing supernatant. It was stored at $-20^{\circ} \mathrm{C}$ until the examination was made. Before SERS measurement, $20 \mu \mathrm{L}$ of serum was mixed with $20 \mu \mathrm{L}$ AgNPs. The mixture is stirred with the pipette on $\mathrm{CaF}_{2}$ slide tip to make it as homogeneous as possible. Then the mixture was incubated for $1 \mathrm{hr}$ at room temperature. Finally, a drop of this mixture was transferred onto an aluminum plate and air dried for SERS measurement (Fig. 1).

Raman spectroscopy. Raman spectra were obtained a Renishaw inVia Raman Spectroscopy with a $785 \mathrm{~nm}$ laser and Charge-Coupled Device (CCD) detector. The acquisition time, laser power and exposure time for all measurements were $15 \mathrm{sec}, 5 \mathrm{~mW}$ and $1 \mathrm{sec}$, respectively. 
Baseline correction was performed for all measurements.

Data acquisition, preprocessing, analysis, and plotting. Data were acquired using the WiRE 3.2 software (Renishaw plc, Wotton-under-Edge, UK). Spectra preprocessing (i.e., fourth polynomial baseline correction and vector normalization), analysis, and plotting were performed using MATLAB ${ }^{\circ}$ (Matlab 7.13, The Mathworks, Natick, MA, USA) Program and analyzed using MATLAB users PLS Toolbox (Eigenvector Research Inc., Wenatchee, WA, USA). Spectral range was set to $3,200 \sim 400 \mathrm{~cm}^{-1}$ on the earlier stages of our studies. Spectral range 920 820 $\mathrm{cm}^{-1}$ was chosen for PLS method on the later stages of method development.

Application of the PLS method. With the development of chemical instruments and processes, experimental design, calibrating instruments and analyzing the data obtained, advanced methods were needed. Chemometrics can be used to improve the development of new methods and to ensure that statistical models are routinely used for data analysis (15).

Raman spectroscopy provides analytical data in a very short period of time. Therefore, it is necessary to use chemometric techniques in order to make the raw data which can be characterized by multiple variables to be meaningful. Chemometric techniques are used to identify, understand, and predict the vast data and have two parts in order to make quantitative and qualitative analysis of the data obtained from experimental studies, and these techniques are also known as linear methods and vary in applicability (16).

In this study, the PLS method which is one of the chemometric techniques was used. To apply PLS method, $920 \sim 820 \mathrm{~cm}^{-1}$ band interval was chosen and the spectral changes of the observed concentrations statistically associated with each other. For this reason, quantitative analysis of ethanol in blood samples was found by applying PLS method to the data obtained from Raman spectroscopy.

According to PLS method, hidden variables obtained for the two data blocks generated by the independent $\mathrm{X}$ variables and the dependent $Y$ variables. The relationship between these two data blocks was investigated by aforementioned hidden variables. In this calculation method, specific steps were performed in the PLS method in order to find the analytes to be investigated. The applied steps are; 10 blood samples (the quantity of ethanol measured by GC) were prepared with AgNPs (Table 1). The characteristics of people using alcohol as body weight, age, gender, and so on were not evaluated statistically. A total of $10 \times 87$ numerical data was obtained from 920 to $820 \mathrm{~cm}^{-1}$ band by SERS method and all data were imported into PLS Toolbox. Cross Validation method in the MATLAB program was chosen. After the PLS model was created and calibrated, the test data to be examined was selected.
Table 1. Quantities of ethanol measured by gas chromatography in blood samples

\begin{tabular}{ll}
\hline \hline Classification & Quantities of ethanol \\
\hline g1 & $386.8 \mathrm{mg} / \mathrm{dL}$ \\
g2 & $382 \mathrm{mg} / \mathrm{dL}$ \\
g3 & $319.6 \mathrm{mg} / \mathrm{dL}$ \\
g4 & $318 \mathrm{mg} / \mathrm{dL}$ \\
g5 & $288 \mathrm{mg} / \mathrm{dL}$ \\
g6 & $274 \mathrm{mg} / \mathrm{dL}$ \\
g7 & $263 \mathrm{mg} / \mathrm{dL}$ \\
g8 & $253.7 \mathrm{mg} / \mathrm{dL}$ \\
g9 & $250 \mathrm{mg} / \mathrm{dL}$ \\
g10 & $232 \mathrm{mg} / \mathrm{dL}$ \\
\hline
\end{tabular}

A total $40 \times 87$ data from 40 blood samples was used to determine the quantity of ethanol. In analyzing the quantity of ethanol in blood samples, the characteristics such as age, gender and weight of the persons were not taken into consideration.

\section{RESULTS}

Characterization of AgNPs. SEM and UV/Visible spectrometry were used for characterization of AgNPs. Obtained images were shown in Fig. 2. AgNPs seems to be spherical and maximum absorbance values were obtained at about $420 \mathrm{~nm}$ for AgNPs (Fig. 2).

Due to the SPR of the nanoparticles, they exhibit intense absorption in the UV/Visible spectrometer. For this reason, UV/Visible spectrometry is the most widely used technique for the characterization of AgNPs.

Qualitative analysis of ethanol by PLS method in blood examined with SERS method. Raman spectrum of pure ethanol was taken for qualitative and quantitative

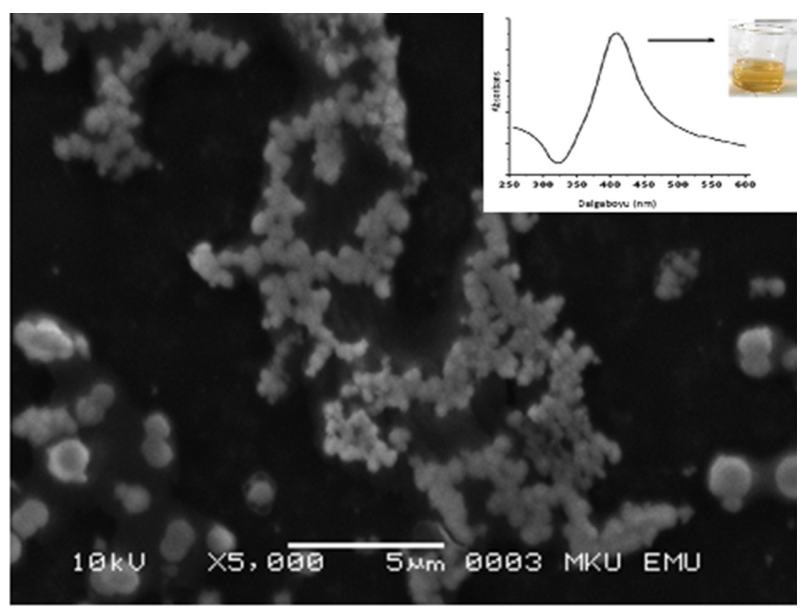

Fig. 2. Images of SEM and UV/Visible spectrometry for AgNPs. 


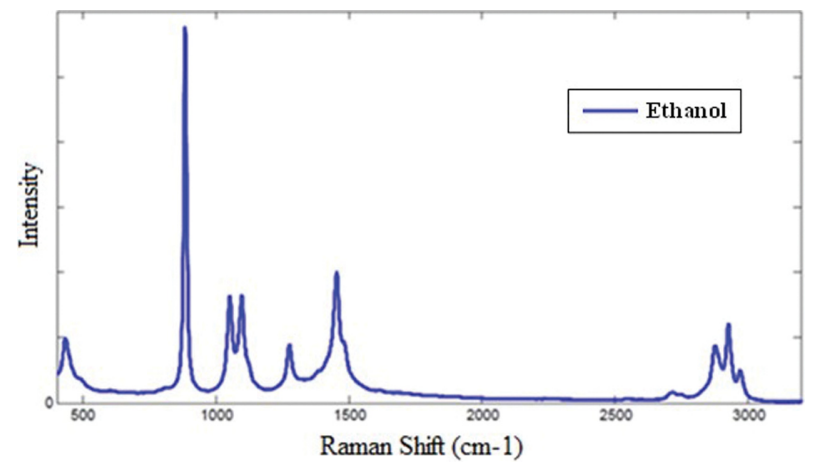

Fig. 3. Raman spectrum of ethanol. The most prominent band belonging to ethanol (C-C stretching) was observed at $882 \mathrm{~cm}^{-1}$.

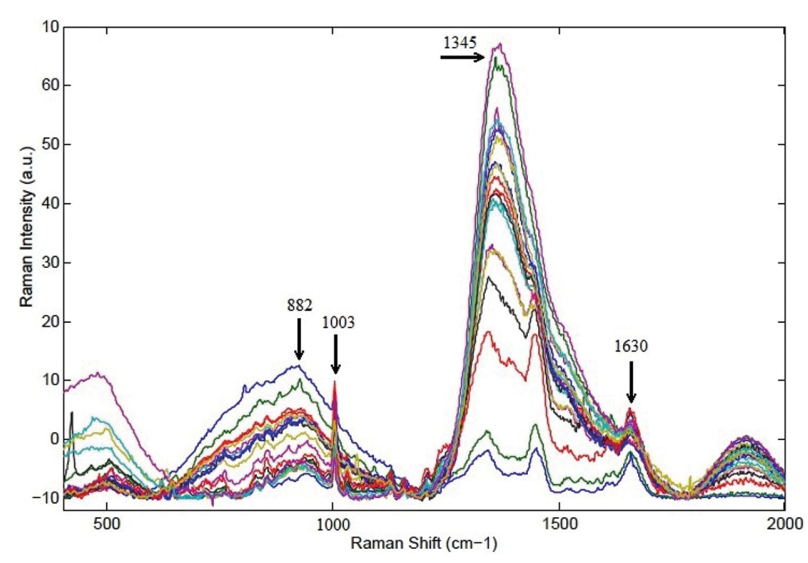

Fig. 4. SERS spectra of blood samples taken from only alcohol users. When the spectra were examined, it was observed that all the peaks were almost the same but their intensity changed. The most prominent band belonging to phenylalanine, tryptophan and protein which is among the structural molecules contained in the blood was observed at 1,003, $1,345,1,630 \mathrm{~cm}^{-1}$, respectively. In blood samples of alcohol users, a band of ethanol was detected at $882 \mathrm{~cm}^{-1}$. Excitation wavelength was at $785 \mathrm{~nm}$, acquisition time was $15 \mathrm{sec}$, and laser power at $5 \mathrm{~mW}$ for all spectra.

analysis ethanol in blood samples. The most prominent band belonging to ethanol (C-C stretching) was observed at $882 \mathrm{~cm}^{-1}$ (Fig. 3).

SERS spectra were obtained using AgNPs from blood samples taken from people who used alcohol (Fig. 4). PLS method was applied as a chemometric method for the quantitative determination of ethanol according to the data obtained from the SERS spectra. In this study, SERS spectra were obtained using AgNPs. The most prominent band belonging to phenylalanine, tryptophan and protein which is among the structural molecules contained in the blood was observed at $1,003,1,345,1,630 \mathrm{~cm}^{-1}$, respectively. In blood samples of alcohol users, a band of ethanol was detected at $882 \mathrm{~cm}^{-1}$ (Fig. 4).

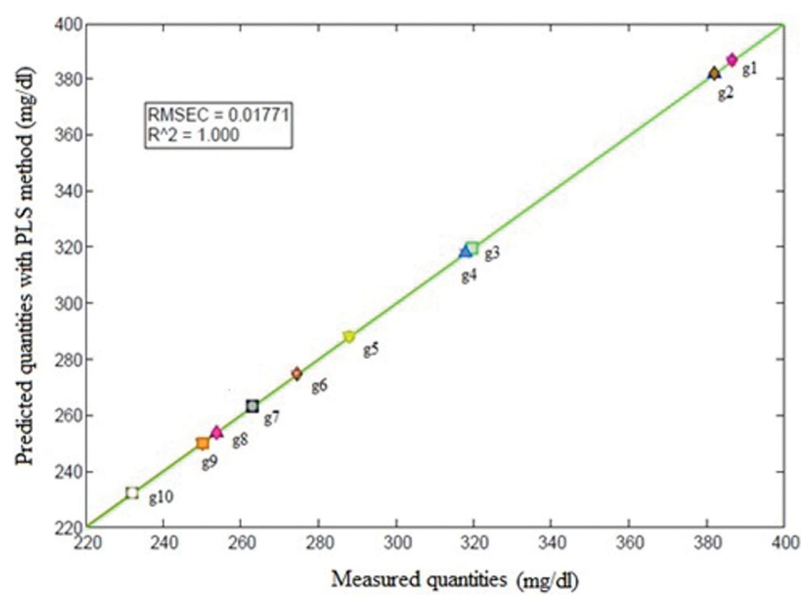

Fig. 5. Calibration curve obtained by PLS method. Ten blood samples, measured by the Gas Chromatography method and detected in high levels of ethanol (ranged from 232 to $386.8 \mathrm{mg} / \mathrm{dL}$ ), were used for calibration. The known values and the values predicted by the PLS method were found to be in harmony.

Quantitative analysis of ethanol in blood samples.

First, a model was established to determine the quantity of ethanol in the blood samples. Second, different blood samples were examined with this calibration model. The blood samples were examined according to this model and the quantity of ethanol was determined. A strong relationship is observed between known concentration values and the values obtained by PLS method $\left(R^{2}=1\right)$ (Fig. 5).

In this study, the spectra of the blood samples which were known to use alcohol and the quantities of ethanol were determined by GC method were obtained by SERS method and the peak changes which it should belong to ethanol were shown. The PLS model was performed using the cross-validation method and quantification was performed. The quantity of ethanol in 20 blood samples from alcohol users was determined. When the blood samples of people using or not using alcohol were compared with the control group according to PLS method, ethanol was determined only in blood samples taken from alcohol users (Fig. 6). As a result, the quantity of ethanol in blood can be easily obtained with the PLS method. However, SERS method can be used as a faster and more sensitive method in routine analyzes in medical science. Also, the quantity of ethanol concentration according to the PLS method was obtained as well (Fig. 6). On the Y or cut-off line those above the zero point was indicated alcoholic blood samples and below that point was indicated non-alcoholic blood samples. The cut-off value according to the PLS method was taken as zero. Those who did not take alcohol above the cut-off value were shown below this value. So, alcoholic bloods were detected positively to PLS method. It 


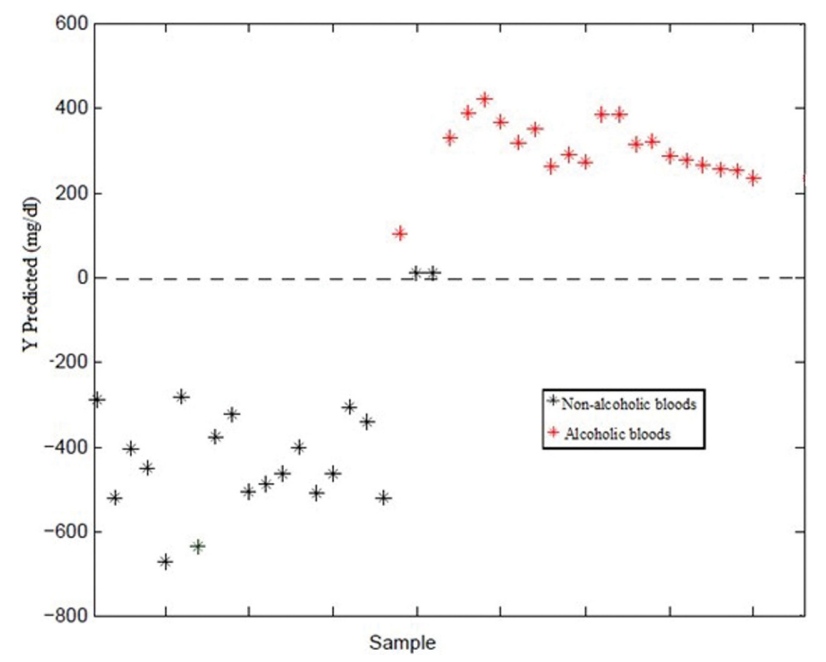

Fig. 6. Comparison of the quantity of ethanol in the blood of people using and not using alcohol with the PLS method. On the $\mathrm{Y}$ or cut-off line those above the zero point was indicated alcoholic bloods and below that point was indicated non-alcoholic bloods. Alcoholic bloods were detected positively to PLS method.

was determined from the obtained data that the quantity of ethanol was higher in 7 people. The others are also close to these values and the quantity determination is as shown in Fig. 6. The blood samples of 2 people who were known to not use alcohol but were addicted to drugs was examined by PLS method was determined to be approximately zero line according to the data obtained. As a result, it was observed that there is a difference between people who are addicted to drugs but who do not use alcohol, people who do not use alcohol and who are not addicted to drugs.

\section{DISCUSSION}

Raman spectroscopy has become increasingly important in recent years, for the rapid and non-destructive analysis. Raman spectroscopy has gained increasing popularity in medical science, and has been used in many studies, such as the identification of human body fluids, to distinguish disease or cell surface antigens (17-20).

There are different screening and confirmatory tests which are still in use to identify and confirm body fluids. These tests are distinguished by their specificity and level of confidence in the identification of body fluids. Molecular methods have been used more recently as the most reliable and sensitive techniques for the identification of body fluids. However, these methods have disadvantages such as difficulty in DNA/RNA extraction, complicated procedure and high cost. Raman spectroscopy allows nondestructive, rapid, specific and low cost identification of body fluids (21).
The fact that the spectra from biological samples can be interpreted for the presence or absence of functional groups and that the reference spectra is comparable to the library database also provides a distinct advantage (22).

There are many studies in the literature concerning blood samples, Raman spectroscopy and chemometric analysis (23). In a study conducted by Virkler et al. (19) has been shown that the Raman spectra obtained from the blood samples are not strongly bound to the donor and that the spectra has two main components. They obtained peaks at $1,000,1,368,1,542$ and $1,620 \mathrm{~cm}^{-1}$ for hemoglobin which is the most important component and contributes to $95 \%$ of the dried weight of red blood cells. They also showed peaks for fibrin, which is the second component, at 967 , $1,248,1,342$ and $1,575 \mathrm{~cm}^{-1}$. They pointed out Raman peaks at $1,000 \mathrm{~cm}^{-1}$ is connected to the presence of phenylalanine in albumin.

Ryder et al. (24) investigated cocaine, heroin, and MDMA in narcotic mixtures at $1,100 \sim 450 \mathrm{~cm}^{-1}$ by microraman spectroscopy. They used different chemometric methods to detect each substance. They showed 4 different Raman peaks from $900 \mathrm{~cm}^{-1}$ to $700 \mathrm{~cm}^{-1}$ as MDMA bands. As a result, they concluded that cocaine, heroin and MDMA can be determined in mixtures. Pérez-Ponce et al. (25) analyzed ethanol and methanol concentrations by FTIR and applied the PLS method to the obtained outputs. They selected a spectra region of $1,100 \sim 1,000 \mathrm{~cm}^{-1}$ for the PLS method. As a result, they stated that the PLS method gives better results than a proportional equations approach. Mobili et al. (26) analyzed the Raman spectra of different species of microorganisms collected by using the PCA and PLS methods. For the chemometric analyzes, they selected spectral regions ranging from 1,500 to $1,700 \mathrm{~cm}^{-1}$ from the spectra obtained by Raman spectroscopy. They have shown that the distinction and classification of certain types of microorganisms can be done thanks to the method they have developed and applied. Bankabur et al. (27) obtained Raman spectra of red and white blood cells under physiological conditions. They have interpreted the Raman peaks that they have obtained from the captured spectra. They have shown that they come to the conclusion efficiently by interpretation Raman spectra of single granulocytes and lymphocytes on the basis of standard protein and nucleic acid vibrational spectroscopy data.

Biological products such as blood products have complex compositions and structures (28). Especially in biological samples, SERS method provides a great advantage in terms of substance analysis and the use of nanoparticles allow enhanced of the surface, resulting in a dense SERS signal.

Received December 19, 2017; Revised February 27, 2018; Accepted March 12, 2018 


\section{REFERENCES}

1. Lachenmeier, D.W, Kanteres, F. and Rehm, J. (2014) Alcoholic beverage strength discrimination by taste may have an upper threshold. Alcohol. Clin. Exp. Res., 38, 2460-2467.

2. Rehm, J., Kanteres, F. and Lachenmeier, D.W. (2010) Unrecorded consumption, quality of alcohol and health consequences. Drug Alcohol. Rev., 29, 426-436.

3. Corrao, G., Bagnardi, V., Zambon, A. and Vecchia, C.L. (2004) A meta-analysis of alcohol consumption and the risk of 15 diseases. Prev. Med., 38, 613-619.

4. Macchia, T., Mancinelli, R., Gentili, S., Lugaresi, E.C, Raponi, A. and Taggi, F. (1995) Ethanol in biological fluids: headspace GC measurement. J. Anal. Toxicol., 19, 241-246.

5. Dawson, D., Li, T. and Grant, B. (2008) A prospective study of risk drinking: at risk for what? Drug Alcohol. Depend., 95, 62-72.

6. Das, R.S. and Agrawal, Y.K. (2011) Raman spectroscopy: recent advancements, techniques and applications. Vib. Spectrosc., 57, 163-176.

7. Triplett, J.S., Hatfield, J.A, Kaeff, T.L., Ramsey, C.R., Robinson, S.D. and Standifer, A.F. (2013) Raman spectroscopy as a simple, rapid, nondestructive screening test for methamphetamine in clandestine laboratory liquids. J. Forensic Sci., 58, 1607-1614.

8. Grasselli, J. (1981) Chemical Applications of Raman Spectroscopy. John Wiley \& Sons, New York.

9. Virkler, K. and Lednev, I.K. (2009) Analysis of body fluids for forensic purposes: from laboratory testing to non-destructive rapid confirmatory identification at a crime scene. Forensic Sci. Int., 188, 1-17.

10. Chalmers, J.M., Edwards, H.G.M. and Hargreaves, M.D. (2012) Infrared and raman spectroscopy in forensic science (1st edition) (Chalmers, J.M., Edwards, H.G.M. and Hargreaves, M.D. Eds.). John Wiley \& Sons, Ltd. pp. 6.

11. Tan, K.S. and Cheong, K.Y. (2013) Advances of Ag, Cu, and $\mathrm{Ag}-\mathrm{Cu}$ alloy nanoparticles synthesized via chemical reduction route. J. Nanopart. Res., 15, 1537.

12. Eustis, S. and El-Sayed, M.A. (2006) Why gold nanoparticles are more precious than pretty gold: noble metal surface plasmon resonance and its enhancement of the radiative and nonradiative properties of nanocrystals of different shapes. Chem. Soc. Rev., 35, 209-217.

13. Sharma, B., Cardinal, M.F., Kleinman, S.L., Greeneltch, N.G., Frontiera, R.R., Blaber, M.G., Schatz, G.C. and Duyne, R.P.V. (2013) High-performance SERS substrates: advances and challenges. MRS Bull., 38, 615-624.

14. Lee, P.C. and Meisel, D. (1982) Adsorption and surfaceenhanced raman of dyes on silver and gold sols. J. Phys. Chem., 86, 3391-3395.

15. Hopke, P.K. (2003) The evolution of chemometrics. Anal. Chim. Acta, 500, 365-377.

16. Shah, R.B., Tawakkul, M.A. and Khan, M.A. (2007) Pro- cess analytical technology: chemometric analysis of Raman and near infra-red spectroscopic data for predicting physical properties of extended release matrix tablets. J. Pharm. Sci., 96, 1356-1365.

17. Sikirzhytskaya, A., Sikirzhytski, V., McLaughlin, G. and Lednev, I.K. (2013) Forensic identification of blood in the presence of contaminations using Raman microspectroscopy coupled with advanced statistics: effect of sand, dust, and soil. J. Forensic Sci., 58,1141-1148.

18. Sikirzhytski, V., Virkler, K. and Lednev, I.K. (2010) Discriminant analysis of Raman spectra for body fluid identification for forensic purposes. Sensors, 10, 2869-2884.

19. Virkler, K. and Lednev, I.K. (2010) Raman spectroscopic signature of blood and its potential application to forensic body fluid identification. Anal. Bioanal. Chem., 396, 525534.

20. An, J.H, Shin, K.J, Yang, W.I. and Lee, H.Y. (2012) Body fluid identification in forensics. BMB Rep., 45, 545-553.

21. Zapata, F., Gregório, I. and García-Ruiz, C. (2015) Body fluids and spectroscopic techniques in forensics: a perfect match? J. Forensic Med., 1, 101.

22. Hayward, I.P., Kirkbride, T.E., Batchelder, D.N. and Lacey, R.J. (1995) Use of a fiber optic probe for the detection and identification of explosive materials by Raman spectroscopy. J. Forensic Sci., 40, 883-884.

23. Açıkgöz, G. (2017) Investigation of Raman spectroscopy and surface enhanced Raman spectroscopy methods of advantages in forensic medicine applications. Ph.D. thesis, Department of Bioengineering and Sciences, Kahramanmaraş Sütçü İmam University, Turkey.

24. Ryder, A.G., O’Connor, G.M. and Glynn, T.J. (1999) Identifications and quantitative measurements of narcotics in solid mixtures using near-IR Raman spectroscopy and multivariate analysis. J. Forensic Sci., 44, 1013-1019.

25. Pérez-Ponce, A., Rambla, F.J, Garrigues, J.M, Garrigues, S. and Guardia, M. (1998) Partial least-squares-Fourier transform infrared spectrometric determination of methanol and ethanol by vapour-phase generation. Analyst, 123, 12531258.

26. Mobili, P., Londero, A., Antoni, G.D. and Gómez-Zavaglia, A. (2010) Multivariate analysis of Raman spectra applied to microbiology: discrimination of microorganisms at the species level. Rev. Mex. Fis., 56, 378-385.

27. Bankapur, A., Zachariah, E., Chidangil, S., Valiathan, M. and Mathur, D. (2010) Raman tweezers spectroscopy of live, single red and white blood cells. PLOS ONE, 5, e10427.

28. Park, T.J., Choi, C.W., Oh, H.K., Kim, J.O., Kim, B.K., Kang, H.K., Kwon, E.J., Gweon, E.J., Park, S.J., Kang, H.I., Jung, K.K., Park, S.M., Kim, J.H., Han, K.W. and Jeong, J.Y. (2017) Stability evaluation of national reference standards for blood Products in Korea. Toxicol. Res., 33, 225231. 